\title{
Do We Need to Consider Ethno-cultural Variation in the Use of Atypical Antipsychotics for Asian Patients with Major Depressive Disorder?
}

\author{
Changsu Han · Chi-Un Pae
}

Published online: 25 May 2013

(c) Springer International Publishing Switzerland 2013

\begin{abstract}
Asian and western countries differ in the prevalence, symptom manifestation, diagnostic procedures, patient recognition and treatments of major depressive disorder (MDD), according to a number of studies. Ethnic differences in pharmacological profiles are also important in the prescription of certain antipsychotic medications because they may impact treatment outcomes and adverse events. Differential pharmacokinetic and pharmacodynamic properties of antipsychotics may be practically useful in the control of specific depressive symptoms. Furthermore, patient compliance with prescribed medications has been found to be different across races and ethnicities. Therefore, this article explores practical clinical issues for the use of atypical antipsychotics in patients with MDD, focusing on ethno-cultural differences.
\end{abstract}

Previous Presentation: Part of the information presented in this article was presented at the round table discussion of psychiatrists from Asia and the USA, sponsored by Korea Otsuka International Asia and Arab.

C. Han

Department of Psychiatry,

Korea University College of Medicine, Seoul, Korea

C.-U. Pae $(\bowtie)$

Department of Psychiatry, Bucheon St Mary's Hospital,

The Catholic University of Korea College of Medicine,

2 Sosa-Dong, Wonmi-Gu, Bucheon,

Kyounggi-Do, 420-717 Seoul, Republic of Korea

e-mail: pae@catholic.ac.kr

C.-U. Pae

Department of Psychiatry and Behavioral Sciences,

Duke University Medical Center, Durham, NC, USA

\section{Diversity in Recognition, Manifestation, Diagnosis and Treatment Issues across Ethnicity and Race}

Substantial differences have been reported in the prevalence, symptom manifestation, developmental mechanisms, patients' recognition and treatment of major depressive disorder (MDD) across different ethnicities [1]. For instance, the prevalence of lifetime MDD in east Asia was less than $3 \%$, versus $16 \%$ in the USA; however, according to a World Health Organization report, the suicide rate in Korea is the highest (31.2/100,000 population and one out of five in MDD patients) among developed countries, which is in contrast to the findings of other prevalence studies, suggesting hidden underlying mental health problems, including MDD [1, 2]. The remarkably low prevalence rates of depression in the Asian population may arise from study methodologies, cultural backgrounds in thinking about emotional distress and MDD symptoms, fear of being stigmatized by addressing mental disorders, or the protective effects of family and social support. According to a recent large epidemiological study in Korea, only $10 \%$ of subjects with clinical depression were found to receive any form of treatment for depression, which is lower than in western countries [3]. Treatment compliance is also substantially different across races and ethnicity. Therefore, it is essential to screen patients properly for symptoms of MDD. Considering the very busy clinical practices in Asia due to problematical national insurance systems, the use of brief self-rating scales of MDD is recommended to evaluate the severity of depressive symptoms $[4,5]$.

Interestingly, Asian and western countries have different profiles of depressive symptoms: Asian MDD patients present as more agitated, anxious and irritable, with lower positive affect and widespread somatic symptoms, while 
western patients show more affective symptoms than physical symptoms [4]. These differences in MDD symptom manifestation have been consistently reported in a number of studies [4]. These trends were strongly replicated in a recent Asian collaborative study investigating the frequency of somatic symptoms in six countries (frequency range of somatic symptoms 35.1-73.3\%) [6].

Consideration of different pharmacodynamic profiles, including affinity on various neurotransmitters and monoamine receptors among each atypical antipsychotic (AA), should also be beneficial for controlling such florid symptoms of MDD when current antidepressants are not adequately effective, especially concerning patient clinical status [7]. According to a recent pooled analysis, quetiapine significantly reduced the symptoms of generalized anxiety disorder, with improvements beginning from week 1; quetiapine also has a favourable profile on sleep disturbance for MDD patients [8]. Quetiapine augmentation may be preferable for MDD patients who are agitated, anxious and with poor sleep quality. A low dose $(2.5 \mathrm{mg} /$ day $)$ of aripiprazole augmentation with antidepressants was also effective, showing a significant improvement of somatic symptoms and psychomotor retardation in patients with MDD [9].

Learning more about the differential experience and manifestation of MDD across ethnic and racial groups will help clinicians evaluate, diagnose and treat MDD more efficiently and effectively [4]. Ethno-cultural modifications in the evaluation and treatment of MDD should also be considered to reflect their cultural diversity maximally.

\section{Clinical Points for the Use of Atypical Antipsychotics for Major Depressive Disorder Regarding Ethnic Differences}

According to a recent meta-analysis [10], adjunctive AAs were significantly more effective in terms of response and remission than placebo. However, rates of discontinuation due to adverse events (AEs) were as much as four times higher for AAs than for placebo. In addition, there were no differences in efficacy and discontinuation rates across AAs, but the profile of major AEs may be substantially different for different AAs. It is well known that compliance to antidepressant therapy is significantly reduced by AEs at an early treatment stage. It is well known that there are substantial pharmacokinetic variations in the metabolism of AAs between Asian and Caucasian populations [11, 12]. In particular, combinations of AAs and antidepressants may increase the risk of developing drug-drug interactions, which are associated with AEs [13]. The cytochrome P450 (CYP) 2D6 and genetic polymorphisms for this CYP isoenzyme system are quite different between Asian and western countries [13]. The overall lower CYP2D6 activity has been consistently observed in east Asians due to the higher variant allele CYP2D6*10 leading to alteration of the enzyme activity, resulting in a lower capacity to metabolize certain drugs that are substrates of CYP2D6, such as antidepressants and antipsychotics [14-16]. Therefore, as fluoxetine and paroxetine are both inhibitors of CYP2D6, they are likely to increase aripiprazole plasma levels. In addition, major enzyme activity depends on the medication dose (e.g. differential enzyme involvement in drug metabolism: CYP3A4 for a high dose of haloperidol and CYP2D6 for a lower dose). The CYP2C19 polymorphism has also to be considered because it is one of the most important polymorphisms in the Asian population. It may affect dosing of citalopram, escitalopram and also sertraline (CYP3A4). The lower activity is related to the presence of the CYP2C19*3 allele that is relatively common in Asian individuals, and which is scarce in other major ethnic groups [14]. The frequency of CYP2D6 variants based on ethnicity has been summarized in Table 1 [17].

How should clinicians adjust doses of AAs for Asian MDD patients? Although there is no clear evidence indicating a dose-response relationship in most antipsychotic augmentation studies, tolerability issues are proportionally increased with dose increment. However, MDD response rates were significantly higher with adjunctive quetiapine XR $300 \mathrm{mg} /$ day (but not $150 \mathrm{mg} /$ day) than with an antidepressant plus placebo in quetiapine XR augmentation studies among the US population [18]. The NNT to achieve an additional response over antidepressant plus placebo were 11-18 and eight to nine in quetiapine XR $150 \mathrm{mg} / \mathrm{day}$ and $300 \mathrm{mg} /$ day, respectively [18]. This indicates that increasing the AA dose is still a viable option for inadequate responders at the initial dose.

Table 1 The frequency of selected CYP450 variants based on ethnicity

\begin{tabular}{llll}
\hline & Asian & Caucasian & African \\
\hline CYP2D6*2 & $9-20 \%$ & $25-35 \%$ & $2-45 \%$ \\
CYP2D6*3 & Infrequent & $2 \%$ & Infrequent \\
CYP2D6*4 & $1 \%$ & $12-21 \%$ & $2 \%$ \\
CYP2D6*5 & $6 \%$ & $2-7 \%$ & $4 \%$ \\
CYP2D6*6 & Infrequent & $1 \%$ & Infrequent \\
CYP2D6*9 & $3 \%$ & $2 \%$ & Infrequent \\
CYP2D6*10 & $51 \%$ & $1-2 \%$ & $6 \%$ \\
CYP2C19*3 & $5-10 \%$ & $1 \%$ & $1 \%$ \\
CYP2C19*17 & $5 \%$ & $20 \%$ & $20 \%$ \\
CYP2D6 Poor metabolizer & $1 \%$ & $5-10 \%$ & $1 \%$ \\
CYP2C19 Poor metabolizer & $15-20 \%$ & $3-5 \%$ & $3 \%$ \\
\hline
\end{tabular}

Adapted and modified from Jacobs Levy [17]

CYP cytochrome 
However, currently available data indicate that the minimally effective AA dose is likely to enhance the likelihood of good treatment outcomes in Asian patients. Most recent studies have suggested that the optimal starting and target daily doses of aripiprazole may be between 2-5 and $10-15 \mathrm{mg} / \mathrm{day}$, which appear tolerable for most depressed patients [9, 19-22], in contrast to earlier findings [23-27]. The authors of the aripiprazole registration trials thought that the adjunct aripiprazole for patients with MDD would require a lower dose than they initially considered $[26,27]$. Similarly, a recent controlled trial has found that a low dose of $2.5 \mathrm{mg} /$ day of aripiprazole augmentation was efficacious and tolerable in Taiwanese patients with MDD [9]. In a recent Japanese study, the mean dose of aripiprazole with selective serotonin reuptake inhibitors was also less than $10 \mathrm{mg} / \mathrm{day}$ [21]. In fact, in the first study [24] of aripiprazole in Asian MDD patients (mean starting dose $5.5 \mathrm{mg} /$ day and target dose $10 \mathrm{mg} /$ day) that adopted a relatively high dosing strategy compared to recent trends conducted in the Asian population [9, 20,21], $23 \%$ of subjects experienced extrapyramidal symptoms (EPSs) during the study, whereas no one had EPSs in the low-dose (fixed $2.5 \mathrm{mg} /$ day) Taiwanese study [9]. Given these findings, the optimal dose of AAs for MDD in the Asian population seems to be substantially lower than for Caucasians; the clear establishment of appropriate starting, target and maintenance doses specifically for Asian patients is warranted.

A recent pooled analysis suggests that east Asian patients (e.g. China, Japan and Korea) have significantly greater risks of EPSs than non east Asian patients, indicating a clear interethnic difference in vulnerability to the development of EPSs [5]. Asian populations have also been found to have slower metabolism rates of certain antipsychotics, leading to an increased prevalence of AEs. Intriguingly, however, the prevalence of tardive dyskinesia (TD) is lower in Asian than western populations [28], suggesting that Asian MDD patients may have some advantageous for long-term treatment with AAs. These differences are thought to be from differences in the expression of genetic polymorphisms, such as the dopamine D3 receptor gene (DRD3) and DRD2 that are principally related to the development of movement disorders, although some debate still exists $[29,30]$. On the other hand, Asian patients were found to have greater susceptibility to developing anticholinergic side effects, blood dyscrasia and metabolic abnormalities, including hypertension, diabetes mellitus and weight gain, from antipsychotic treatment [5, 31, 32]. Therefore, clinicians should consider ethnic differences in pharmacokinetics, particularly with regard to the development of AEs, in prescribing AAs.

To treat EPSs in clinical practice, anticholinergic agents are usually prescribed. What is appropriate in the use of anticholinergic agents? (For example, should they be used as a prophylactic, after EPS, or during the entire treatment?) There are no data supporting a prophylactic use of anticholinergic agents for EPSs, and existing research indicates that anticholinergic agents have questionable long-term benefits and carry the risk of various AEs, including cognitive impairment and worsening of movement disorders [33]. Therefore, the use of anticholinergic agents for MDD patients is recommended to be short term with early discontinuation, particularly for Asians with their greater vulnerability to anticholinergic AEs. In fact, the most common interventions associated with EPS resolution were dose reduction of aripiprazole (51\%) and no intervention (36\%), in registration clinical trials of aripiprazole for MDD [7].

Health insurance policies to reduce healthcare utilization and expenditures vary substantially across Asian countries (as well as compared with western countries) depending on their national funding resource [34], and thus

Table 2 Considerations for augmenting antidepressants with atypical antipsychotics for Asian patients with major depressive disorder based on different ethnic backgrounds

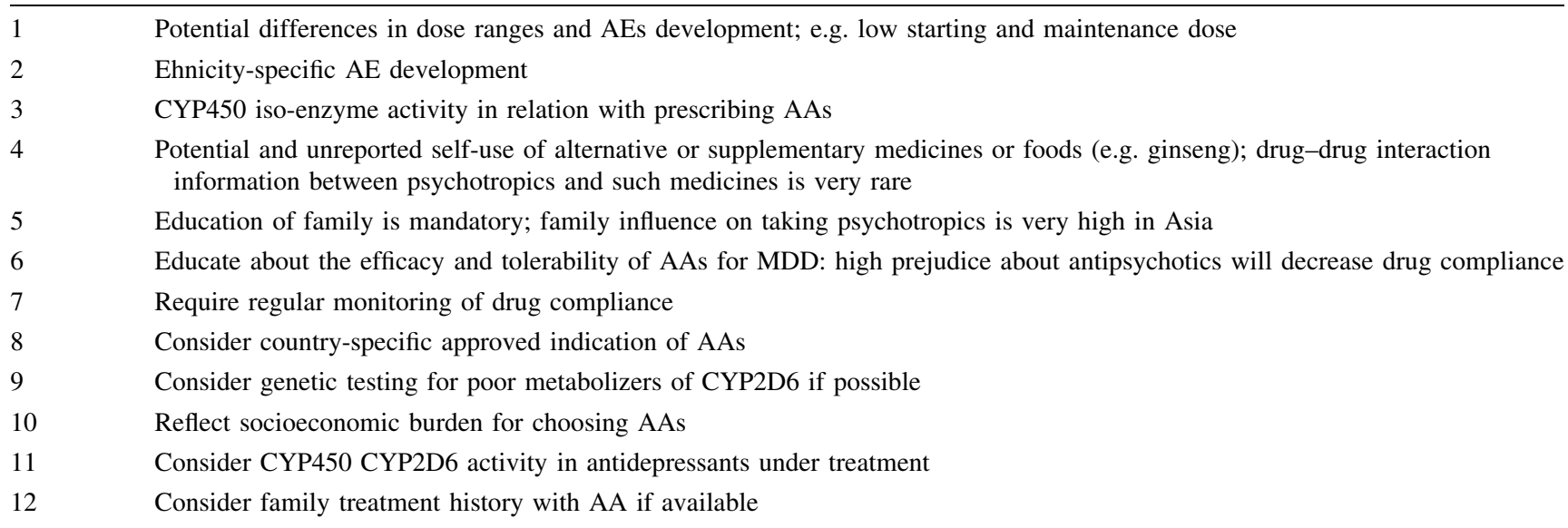

$A A$ atypical antipsychotic, $A E$ adverse event, $C Y P$ cytochrome, $M D D$ major depressive disorder 
cost versus clinical effects for individual AAs may differ in the treatment of MDD [35]. This creates another important consideration in the prescription of AAs for Asian MDD patients.

While interracial pharmacokinetic and pharmacodynamic differences for psychotropic medications are known to impact treatment outcomes, there are not yet definitive data determining the most appropriate use of AAs with MDD patients of different races and ethnicities. More clinical data are needed to expand and better define these influences on best clinical practice.

Therefore, differences in $\mathrm{AE}$ profiles, dose requirements, psychiatric and medical comorbidities, drug interactions, pharmacodynamic differences and approved indications should be considered in the selection of AAs as an augmentation therapy for treating Asian patients with MDD. Recommendations for augmenting antidepressants with AAs for Asian patients with MDD considering different ethnic backgrounds are presented in Table 2.

\section{Conclusions}

As several AAs have demonstrated efficacy as augmentation of antidepressants in patients who fail to show adequate response to initial treatment, the use of AAs has dramatically increased [36]; however, there is insufficient clinical information to develop clear guidelines for the use of AAs in Asian MDD patients. Although AAs have demonstrated efficacy as augmentation therapy for treating MDD in western populations, studies with Asian populations are limited, and those that exist suggest differences. Further controlled clinical trials, long-term studies, analyses of benefit-risk ratios of currently approved AAs with various antidepressants and studies based on pharmacokinetic variations between Asian and western population are greatly needed. The viability of AAs as a treatment option for Asian patients with MDD should depend on further information regarding Asian-specific pharmacological and clinical factors associated with treatment outcomes and tolerability issues in clinical practice.

Acknowledgments Although Korea Otsuka International Asia and Arab (KOIAA) was involved in supporting the production of the supplement, the content of the manuscript, its review and revision, and the decision to submit to CNS Drugs were made solely by the authors and the supplement guest editor.

Sources of Financial Support The authors did not receive honorarium for writing this manuscript.

Conflict of interest Dr Han has received research grants from GlaxoSmithKline Korea, Eisai Korea, Korea Otsuka International Asia and Arab, Hanlim Pharmaceutical, Janssen Pharmaceuticals Korea, Eli Lilly and Company Korea, Korean Health Technology
R\&D Project, Ministry of Health \& Welfare, Republic of Korea, Korean Research Foundation, and Otsuka Korea; and has received honoraria from GlaxoSmithKline Korea, Lundbeck Korea, AstraZeneca Korea, Janssen Pharmaceuticals Korea, Eisai Korea, Abott Korea, Eli Lilly and Company Korea, Norvatis Korea and Otsuka Korea. Dr Pae has received research grants from Eisai Korea, Janssen Pharmaceuticals Korea, Korean Health Technology R\&D Project, Ministry of Health \& Welfare, Republic of Korea, and Korean Research Foundation; and has received honoraria from Pfizer Korea and Otsuka Korea.

Disclosure This manuscript has been published in a journal supplement that was created with an unrestricted educational grant from Korea Otsuka International Asia and Arab (KOIAA).

\section{References}

1. Weissman MM, Bland RC, Canino GJ, Faravelli C, Greenwald S, Hwu HG, et al. Cross-national epidemiology of major depression and bipolar disorder. J Am Med Assoc. 1996;276:293-9.

2. Kessler RC, Berglund P, Demler O, Jin R, Koretz D, Merikangas $\mathrm{KR}$, et al. The epidemiology of major depressive disorder: results from the National Comorbidity Survey Replication (NCS-R). J Am Med Assoc. 2003;289:3095-105.

3. Cho SJ, Lee JY, Hong JP, Lee HB, Cho MJ, Hahm BJ. Mental health service use in a nationwide sample of Korean adults. Soc Psychiatry Psychiatr Epidemiol. 2009;44:943-51.

4. Kalibatseva Z, Leong FT. Depression among Asian Americans: review and recommendations. Depress Res Treat. 2011;2011: 320902.

5. Ormerod S, McDowell SE, Coleman JJ, Ferner RE. Ethnic differences in the risks of adverse reactions to drugs used in the treatment of psychoses and depression: a systematic review and meta-analysis. Drug Saf. 2008;31:597-607.

6. Lee P, Zhang M, Hong JP, Chua HC, Chen KP, Tang SW, et al. Frequency of painful physical symptoms with major depressive disorder in Asia: relationship with disease severity and quality of life. J Clin Psychiatry. 2009;70:83-91.

7. Pae CU, Forbes A, Patkar AA. Aripiprazole as adjunctive therapy for patients with major depressive disorder: overview and implications of clinical trial data. CNS Drugs. 2011;25:109-27.

8. Stein DJ, Bandelow B, Merideth C, Olausson B, Szamosi J, Eriksson H. Efficacy and tolerability of extended release quetiapine fumarate (quetiapine XR) monotherapy in patients with generalised anxiety disorder: an analysis of pooled data from three 8-week placebo-controlled studies. Hum Psychopharmacol. 2011;26:614-28.

9. Lin CH, Lin SH, Jang FL. Adjunctive low-dose aripiprazole with standard-dose sertraline in treating fresh major depressive disorder: a randomized, double-blind, controlled study. J Clin Psychopharmacol. 2011;31:563-8.

10. Nelson JC, Papakostas GI. Atypical antipsychotic augmentation in major depressive disorder: a meta-analysis of placebo-controlled randomized trials. Am J Psychiatry. 2009;166:980-91.

11. Shen H, He MM, Liu H, Wrighton SA, Wang L, Guo B, et al. Comparative metabolic capabilities and inhibitory profiles of CYP2D6.1, CYP2D6.10, and CYP2D6.17. Drug Metab Dispos: Biol Fate Chem. 2007;35:1292-300.

12. Chong SA, Tan CH, Khoo YM, Lee HS, Wong KE, Ngui F, et al. Clinical evaluation and plasma clozapine concentrations in Chinese patients with schizophrenia. Ther Drug Monit. 1997;19:219-23.

13. Chen ML. Ethnic or racial differences revisited: impact of dosage regimen and dosage form on pharmacokinetics and pharmacodynamics. Clin Pharmacokinet. 2006;45:957-64. 
14. Bertilsson L. Metabolism of antidepressant and neuroleptic drugs by cytochrome p450 s: clinical and interethnic aspects. Clin Pharmacol Ther. 2007;82:606-9.

15. Dahl ML, Yue QY, Roh HK, Johansson I, Sawe J, Sjoqvist F, et al. Genetic analysis of the CYP2D locus in relation to debrisoquine hydroxylation capacity in Korean, Japanese and Chinese subjects. Pharmacogenetics. 1995;5:159-64.

16. Johansson I, Oscarson M, Yue QY, Bertilsson L, Sjoqvist F, Ingelman-Sundberg M. Genetic analysis of the Chinese cytochrome P4502D locus: characterization of variant CYP2D6 genes present in subjects with diminished capacity for debrisoquine hydroxylation. Mol Pharmacol. 1994;46:452-9.

17. Levy J. Equity management personalized genomic medicine program. Genetics of CYP2D6 and CYP2C19. Drug Response. https://atlantichealth.dnadirect.com/grc/patient-site/psychiatric-drugresponse/genetics-of-cyp2d6-and-cyp2c19-drug-response.html. Accessed 11 Sept 2012.

18. Pae CU, Sohi MS, Seo HJ, Serretti A, Patkar AA, Steffens DC, et al. Quetiapine XR: current status for the treatment of major depressive disorder. Prog Neuropsychopharmacol Biol Psychiatry. 2010;34:1165-73.

19. Simon JS, Nemeroff CB. Aripiprazole augmentation of antidepressants for the treatment of partially responding and nonresponding patients with major depressive disorder. J Clin Psychiatry. 2005;66:1216-20.

20. Chen SJ, Hsiao YL, Shen TW, Chen ST. The effectiveness and safety of adjunctive aripiprazole in Taiwanese patients with antidepressant-refractory major depressive disorder: a prospective, open-label trial. J Clin Psychopharmacol. 2012;32:56-60.

21. Yoshimura R, Kishi T, Hori H, Ikenouchi-Sugita A, Katsuki A, Umene-Nakano W, et al. Comparison of the efficacy between paroxetine and sertraline augmented with aripiprazole in patients with refractory major depressive disorder. Prog Neuropsychopharmacol Biol Psychiatry. 2012;39:355-7.

22. Fava M, Mischoulon D, Iosifescu D, Witte J, Pencina M, Flynn M, et al. A double-blind, placebo-controlled study of aripiprazole adjunctive to antidepressant therapy among depressed outpatients with inadequate response to prior antidepressant therapy (ADAPTA Study). Psychother Psychosom. 2012;81:87-97.

23. Hellerstein DJ, Batchelder S, Hyler S, Arnaout B, Corpuz V, Coram L, et al. Aripiprazole as an adjunctive treatment for refractory unipolar depression. Prog Neuropsychopharmacol Biol Psychiatry. 2008;32:744-50.

24. Pae CU, Patkar AA, Jun TY, Lee C, Masand PS, Paik IH. Aripiprazole augmentation for treatment of patients with inadequate antidepressants response. Depression Anxiety. 2007;24:522-6.

25. Patkar AA, Peindl K, Mago R, Mannelli P, Masand PS. An openlabel, rater-blinded, augmentation study of aripiprazole in treatment-resistant depression. Prim Care Companion J Clin Psychiatry. 2006;8:82-7.

26. Marcus RN, McQuade RD, Carson WH, Hennicken D, Fava M, Simon JS, et al. The efficacy and safety of aripiprazole as adjunctive therapy in major depressive disorder: a second multicenter, randomized, double-blind, placebo-controlled study. J Clin Psychopharmacol. 2008;28:156-65.

27. Berman RM, Marcus RN, Swanink R, McQuade RD, Carson WH, Corey-Lisle PK, et al. The efficacy and safety of aripiprazole as adjunctive therapy in major depressive disorder: a multicenter, randomized, double-blind, placebo-controlled study. J Clin Psychiatry. 2007;68:843-53.

28. Chiu H, Shum P, Lau J, Lam L, Lee S. Prevalence of tardive dyskinesia, tardive dystonia, and respiratory dyskinesia among Chinese psychiatric patients in Hong Kong. Am J Psychiatry. 1992;149:1081-5.

29. Koning JP, Vehof J, Burger H, Wilffert B, Al Hadithy A, Alizadeh $\mathrm{B}$, et al. Association of two DRD2 gene polymorphisms with acute and tardive antipsychotic-induced movement disorders in young Caucasian patients. Psychopharmacology. 2012;219:727-36.

30. Utsunomiya K, Shinkai T, Sakata S, Yamada K, Chen HI, De Luca V, et al. Genetic association between the dopamine D3 receptor gene polymorphism (Ser9Gly) and tardive dyskinesia in patients with schizophrenia: a reevaluation in East Asian populations. Neurosci Lett. 2012;507:52-6.

31. Subramaniam M, Ng C, Chong SA, Mahendran R, Lambert T, Pek E, et al. Metabolic differences between Asian and Caucasian patients on clozapine treatment. Hum Psychopharmacol. 2007;22: $217-22$.

32. Matsuda KT, Cho MC, Lin KM, Smith MW, Young AS, Adams JA. Clozapine dosage, serum levels, efficacy, and side-effect profiles: a comparison of Korean-American and Caucasian patients. Psychopharmacol Bull. 1996;32:253-7.

33. Desmarais JE, Beauclair L, Margolese HC. Anticholinergics in the era of atypical antipsychotics: short-term or long-term treatment? J Psychopharmacol. 2012;26:1167-74.

34. Kwon S. Health care financing in Asia: key issues and challenges. Asia-Pacific J Public Health/Asia-Pacific Acad Consortium Public Health. 2011;23:651-61.

35. Jing Y, Kalsekar I, Curkendall SM, Carls GS, Bagalman E, Forbes RA, et al. Intent-to-treat analysis of health care expenditures of patients treated with atypical antipsychotics as adjunctive therapy in depression. Clin Ther. 2011;33:1246-57.

36. Konstantinidis A, Papageorgiou K, Grohmann R, Horvath A, Engel R, Kasper S. Increase of antipsychotic medication in depressive inpatients from 2000 to 2007: results from the AMSP International Pharmacovigilance Program. Int J Neuropsychopharmacol. 2012;15:449-57. 